\title{
Anti-noise Capability Improvement of Minimum Energy Combination Method for SSVEP Detection
}

\author{
Omar Trigui \\ Wassim Zouch \\ Advanced Technologies for Medicine and Signals 'ATMS', King Abdulaziz University (KAU) Jeddah, Saudi Arabia \\ ENIS, Sfax University, Tunisia \\ Mohamed Ben Messaoud \\ Advanced Technologies for Medicine and Signals 'ATMS', \\ ENIS, Sfax University, Tunisia
}

\begin{abstract}
Minimum energy combination (MEC) is a widely used method for frequency recognition in steady state visual evoked potential based BCI systems. Although it can reach acceptable performances, this method remains sensitive to noise. This paper introduces a new technique for the improvement of the MEC method allowing ameliorating its Anti-noise capability. The Empirical mode decomposition (EMD) and the moving average filter were used to separate noise from relevant signals. The results show that the proposed BCI system has a higher accuracy than systems based on Canonical Correlation Analysis (CCA) or Multivariate Synchronization Index (MSI). In fact, the system achieves an average accuracy of about $99 \%$ using real data measured from five subjects by means of the EPOC EMOTIVE headset with three visual stimuli. Also by using four commands, the system accuracy reaches $91.78 \%$ with an information-transfer rate of about $27.18 \mathrm{bits} / \mathrm{min}$.
\end{abstract}

Keywords- Brain-Computer Interface; Steady State Visual Evoked Potential; Minimum Energy Combination; Empirical Mode Decomposition.

\section{INTRODUCTION}

Severely paralyzed people with neuromuscular disorders lose the majority or the totality of their movement and expression abilities. This is the case of people with locked-in syndrome, Amyotrophic lateral sclerosis and Spinal cord injury. A Brain-Computer Interface (BCI) is a tool for mobility, communication and control assistance which can provide them with the possibility to interact with their surroundings [1]. The principle of a BCI is to detect the brain activity from the scalp and convert it into commands to control devices such as prosthesis and computers. The control is done only by thought without any apparent movement. The electroencephalography (EEG) is usually used in BCI field for brain activity measurements. This is mainly due to its time resolution efficient for real-time applications, its low cost compared to other technics and the possibility to wear an EEG headset everywhere.

The P300 evoked potentials, the Event-Related Synchronization and Desynchronization (ERS/ERD) and the Steady State Visual Evoked Potential (SSVEP) are the most promising EEG brain activity patterns. SSVEPs are nearsinusoidal waveforms from the occipital area reflecting a visual stimulation [2]. SSVEP-based BCI systems offer many advantages: the small number of required electrodes which makes the equipment cheaper, the no need of a tiring training, the suitability for almost any person and any environment and the better resistance face to noise and artifacts compared with other brain responses [3] [4]. Also, the performances reached by these systems are very encouraging. For example, F. Gembler et al. [5] have made an experiment where SSVEP was used to distinguish one among four possible commands. Ten subjects from different age ranges participated in the study and the data were acquired from 8 channels with a sampling rate of $128 \mathrm{~Hz}$. The average accuracy was about 93\% which makes the system functional and useful.

Several processing methods were presented to distinguish the target at which the subject gazes [6]. For instance, the Minimum Energy Combination (MEC) method proposed by O. Friman et al. [7] estimates the signal to noise ratio (SNR) corresponding to the stimuli frequencies then selects the frequency that maximizes this quantity. This method was exploited by N. Chumerin et al. [8] to create an SSVEP-based $\mathrm{BCI}$ game. The task was to navigate an avatar through a maze using four commands. The average accuracy of six subjects was about $82.4 \%$ which is considered acceptable. Moreover, Z. Lin et al. [8] use a frequency recognition method based on the Canonical Correlation Analysis (CCA). The essence of this method is to extract the correlation coefficients between the EEG signal and the reference signals then to select the frequency which maximizes this coefficient. Both MEC and CCA methods offer good performances thanks to the multichannel and multi-harmonics properties. G. Hakvoort et al. [9] emphasize the usefulness of the multi-channel criterion by making a comparison between the CCA as a multi-channel based method and the power spectral density analyses (PSDA) as a mono-channel based one. The average accuracy of seven subjects was $47.81 \%$ using PSDA and 78.12 using CCA in the discrimination among seven different frequencies. This result clearly demonstrates the importance of multi-channel techniques. On the other hand, despite the solid mathematical foundations of the CCA and the MEC methods, a comparison between them shows that the CCA appears to have widely superior performances. For example, N. Mora et al. [10] make an experiment where five subjects are asked to gaze at one of four possible LEDs flickering with different stimulation frequencies. Data is measured from six different channels then processed using different discrimination methods. Results 
show that CCA method ensures a higher accuracy rate by about $13 \%$ than MEC method. In another study results indicate that the high sensitivity to the noise level of MEC method leads to a lower accuracy rate [11].

Recently, Y. Zhang et al. [12] designed a new recognition method based on the multivariate synchronization index (MSI). The idea is to calculate the synchronization indexes reflecting the similarity between the EEG data and reference signals similar to those used in CCA method. Following the same idea, the frequency which maximizes the synchronization index is chosen.

The paper presents a novel amelioration of MEC method for achieving better resistance to noise. The Empirical mode decomposition and the moving average are used to reject irrelevant signals. The remaining signals located at the stimuli and harmonics frequency band are used to recognize the target.

The following section describes the origin of the noise in the SSVEP signal. Then the recognition methods are presented. The new amelioration of MEC method is presented in section four. To validate the improvement of the modified method, a comparison with the three methods is carried out. Finally, section 6 concludes the paper.

\section{SSVEP AND NOISE SOURCES}

The SSVEP occurs when a subject gazes at a light source target flickering with a fixed frequency. It can be detected as a signal power increase at the same frequency of the stimulus. In addition, a number of harmonic frequencies multiple of the principal frequency can be detected [13]. To exploit this phenomenon, several targets are presented in front of the subject. Each target flickers with a unique frequency. Besides one command is assigned to each target. To execute a command, the subject has to gaze directly at the appropriate target. Due to the effect of cortical magnification, the quality of SSVEP increases if the subject gazes directly at a stimulus object located in the center of his vision field [14] [15]. Likewise, the retinal cones distribution shows that the foveola located in the center of the visual field is more sensitive to the light. Therefore, as it was proven by A. González-Mendoza et al. [16], the amplitude of the SSVEP increases proportionally with the area size of the visual stimulus. Consequently, the noise level increases if the stimulus does not exist in the center of the vision field or if the light intensity is not sufficient. Also, the choice of a wrong stimulus color can weaken the power of the SSVEP response [17]. Before performing ameliorations on the MEC method, the stimulus parameters effect on the performances of the processing methods is studied. A comparison of MEC method versus other ones for different noise levels is investigated.

\section{MATERIALS AND EXPERIMENTS}

Five healthy male volunteers participated in the study. Their ages are 28, 30, 30, 34, and 58. None of the subjects had visual or neurological disorders or a previous experience with the BCI systems. Subjects were asked to sit in a comfortable chair in a room with low noise and luminance level and to avoid any movement. The Epoc Emotiv headset was used to acquire the EEG signal from the scalp. Data is sampled at 128 $\mathrm{Hz}$ within a bandwidth from 0.2 to $45 \mathrm{~Hz}$ with a digital notch filter at $50 \mathrm{~Hz}$ and $60 \mathrm{~Hz}$. The choice of this equipment is due to its short preparation time and low price which are important factors to bring BCI systems into daily life. Furthermore, Epoc Emotiv headset had shown good performances in SSVEP based BCI as in Y. Liu et al. [18] study where the accuracy rate reached $95.83 \pm 3.59 \%$ with online application. To cover the maximum zone of the occipital area, data were obtained from electrodes T7, T8, P7, P8, O1, and O2 according to the 10/20 international system. In the present study, the chosen application is the wheelchair navigation command. In the first experiment, 3 commands are used: one to move forward, one to turn left, and one to turn right. Thus, the stimulation system is composed of three LEDs positioned on the left, top, and right sides of a computer screen. The LEDs are flickering with fixed frequencies: 8,9 , and $10 \mathrm{~Hz}$ respectively and the subject sits $0.6 \mathrm{~m}$ far from them. In the second experiment, in order to improve the information-transfer rate of the system, another command is added to the system allowing to move backward. Its appropriate target which flickers at $11 \mathrm{~Hz}$ is positioned at the bottom side of the screen. To ensure the control of the stimulation frequencies with precision, an electronically device based on the STM8 microcontroller was used. Subjects were asked to follow the scenario of the figure 1 .

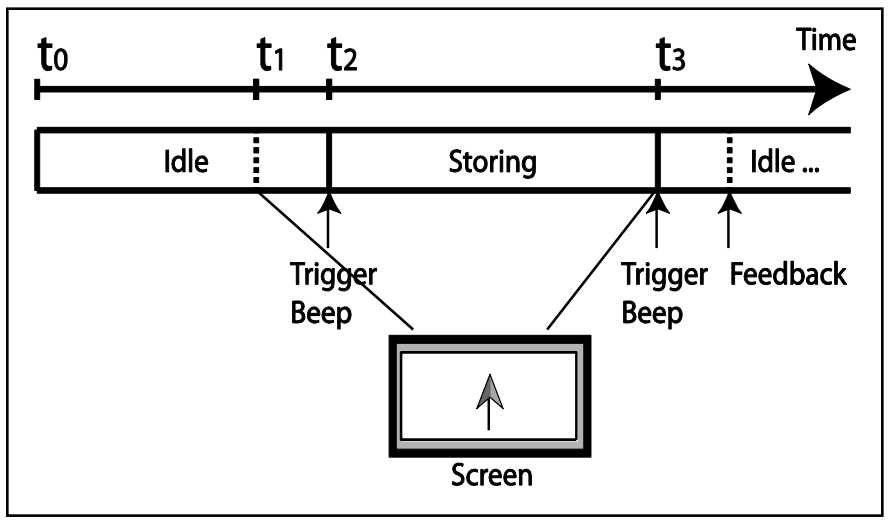

Fig. 1. Experimental Scenarios.

The experiment consists of 18 trials. Each one lasts between 3 and 8 seconds. The first period is an idle one where EEG signals are not used. At $t=t_{1}$ a beep sound indicates the beginning of the trial accompanied by an arrow pointing the LED to gaze at. At $t=t_{2}$ data start to be sent to the processing bloc. At $t=t_{3}$ another beep sound triggers indicating the end of the trial. The feedback is shown on the screen as an increment in the value of true or false trials counters.

\section{METHODS}

The recognition techniques in SSVEP-based BCIs are based on the estimation of a coefficient that reflects the power of a stimulus frequency in the EEG signal. The frequency that maximizes this coefficient is considered as the frequency of the selected target. 


\section{A. MEC method in the SSVEP-based BCI}

MEC based method uses the SNR as a clue of the stimulation frequency. The diagram of figure 2 shows the different steps of the method.

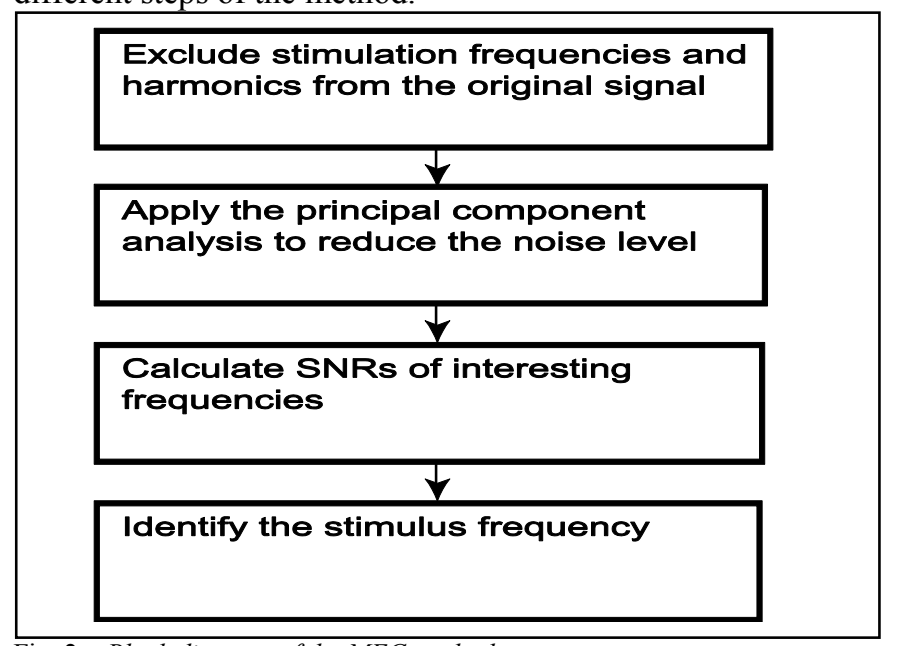

Fig. 2. Block diagram of the MEC method.

The EEG signal $y_{i}(t)$ is represented by a linear model following the equation (1).

$y_{i}(t)=\sum_{k=1}^{N_{h}+1} a_{i, k} \sin \left(2 \pi k f t+\phi_{i, k}\right)+\sum_{j}\left(b_{i, j}+z_{j}(t)\right)+e_{i}(t)$

Where $i$ is the unique electrode identifier, $N_{h}$ is the number of harmonics, and $f$ is the stimulation frequency.

The model is decomposed into the sum of three quantities. The first one defines the frequencies of interest where $a_{i, k}$ and $\phi_{i, k}$ are respectively the specific amplitudes and phases, the second is a nuisance signals $z_{j}(t)$ such as the artifacts where $b_{i, j}$ is the weight factor, and the third quantity represents the noise.

The aim of the two first steps of the block diagram is to reduce the noise level and to increase the interesting frequencies level. First of all, the frequencies of interest are eliminated by projecting the matrix $Y$ of the EEG signal onto the orthogonal complement of the matrix $X$ containing a pair of $\sin (2 \pi k f t)$ and $\cos (2 \pi k f t)$ in its columns.

$$
\ddot{Y}=Y-X\left(X^{T} X\right)^{-1} X^{T} Y
$$

Where

$\ddot{Y}$ is the matrix of uninteresting signals,

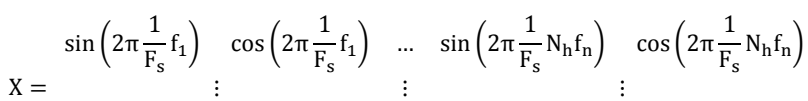

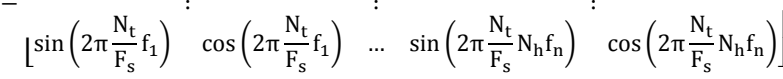

$F_{s}$ is the sampling frequency, $N_{t}$ is the number of samples, and $f_{1} \ldots f_{n}$ are the stimulation frequencies.

In the second step, the principal component analysis (PCA) is utilized to find a linear combination minimizing the variance of the matrix $\ddot{Y}$. The application of this linear combination on the original matrix $Y$ allows the creation of the matrix $S$ with a reduced noise level.

In the following steps, the SNR values, as described in equation 3, are measured then the stimulus frequency that maximizes the SNR is considered as the frequency of interest.

$$
\operatorname{SNR}(f)=P(f) / \sigma(f)
$$

Where $P(f)$ is the signal power function and $\sigma(f)$ is an estimation of the noise power.

\section{B. CCA method in the SSVEP-based BCI}

The CCA allows to compare two groups of variables in order to know if they describe the same phenomenon. In the SSVEP-based BCI, the CCA is used for a comparison between the multi-channel EEG signals and a reference signal $R_{f_{i}}$ including the stimulus frequencies and the harmonics.

$$
R_{f_{i}}=\left(\begin{array}{c}
\sin \left(2 \pi f_{i}(t)\right) \\
\cos \left(2 \pi f_{i}(t)\right) \\
\mathrm{M} \\
\sin \left(2 \pi N_{h} f_{i}(t)\right) \\
\cos \left(2 \pi N_{h} f_{i}(t)\right)
\end{array}\right)
$$

Figure 3 depicts the different steps of the CCA based method. The value of the frequency of interest is the same as the reference frequency that maximizes the correlation coefficient. 


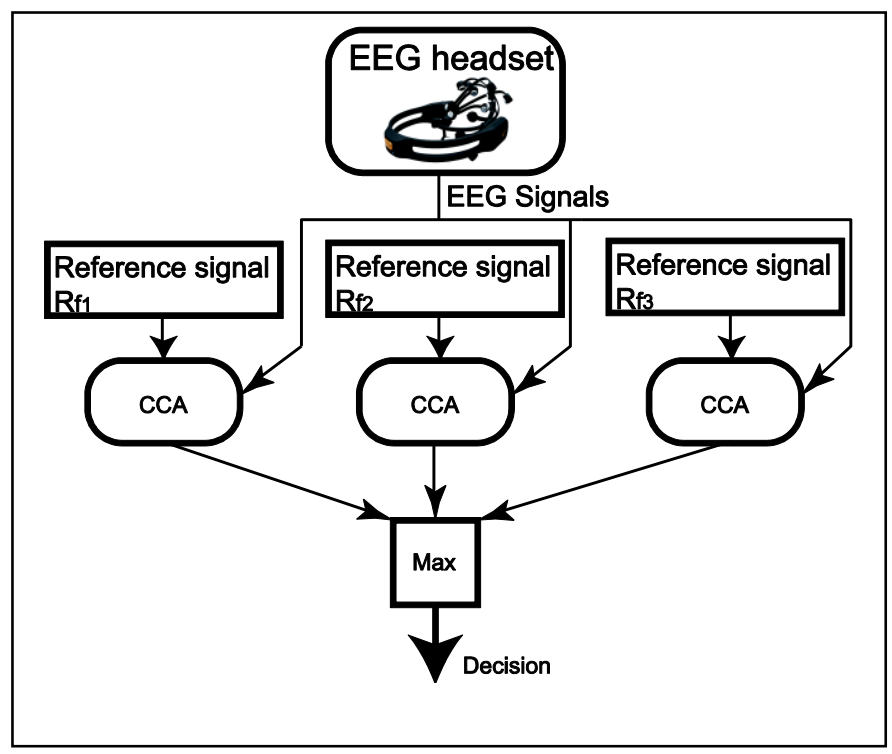

Fig. 3. Block diagram of the CCA method.

\section{MSI method in the SSVEP-based BCI}

The MSI method estimates the synchronization index between the EEG signal $Y$ and the reference signals $R_{f_{i}}$.

The correlation matrix between two sets of data $Y$ and $R$ is given by the equation (5):

$$
C=\left[\begin{array}{ll}
C_{x x} & C_{x y} \\
C_{y x} & C_{y y}
\end{array}\right]
$$

Where:

$$
C_{x x}=\frac{1}{M} Y Y^{T}, C_{x y}=C_{y x}=\frac{1}{M} Y R^{T}, C_{y y}=\frac{1}{M} R R
$$

and $M$ is the number of samples.

To reduce the effect of the autocorrelation, the following linear transformation is applied to the matrix $C$ producing the matrix $\ddot{C}$.

$$
\begin{gathered}
\ddot{\mathrm{C}}=\mathrm{UCU}^{\mathrm{T}} \\
\text { Where: } U=\left[\begin{array}{cc}
C_{x x}{ }^{-\frac{1}{2}} & 0 \\
0 & C_{y y}^{-\frac{1}{2}}
\end{array}\right] .
\end{gathered}
$$

A normalization of the eigenvalues $\lambda_{i}$ of the matrix $\ddot{C}$ is given by:

$$
\lambda_{i}^{\prime}=\frac{\lambda_{i}}{\sum_{j=1}^{P} \lambda_{j}}
$$

Where: $P$ is the number of eigenvalues.

Finally, the synchronization index $S$ is defined by the equation (8).

$$
S=1+\frac{\sum_{i=1}^{P} \lambda_{i}^{\prime} \log \left(\lambda_{i}^{\prime}\right)}{\log (P)}
$$

The $S$ quantity tends towards 0 when $Y$ and $R$ are increasingly uncorrelated and towards 1 when $Y$ and $R$ are increasingly correlated. Consequently, the frequency of the reference which has the maximum synchronization index is considered as the frequency of interest.

\section{MEC method amelioration}

The EEG signals have a poor signal to noise ratio [19] which makes the brain activity patterns difficult to be detected. Nevertheless, it is not always possible to discriminate between the different mental tasks.

The goal of this improvement is to reduce the noise sensitivity of the MEC method. Considering that interesting signals are composed of stimulation frequencies and harmonics, and the rest is noise. The noise sensitivity effect can be caused by the fact that a part of the noise is considered as relevant or a part of the interesting signal is considered as irrelevant. In both cases, the problem is in the separation between the noise and the interesting signal. Thus, this problem can be localized in the first step of the MEC method.

The idea of the improvement is to use the empirical mode decomposition (EMD) to divide the EEG signal into useful and noise signals instead of using the matrix projection.

The EMD was firstly proposed by Huang et al. [20] as an efficient method to evaluate the frequency and amplitude of time-series with excellent time resolution. It divides the original signal into some Intrinsic Mode Functions (IMF), which are different scales of oscillation components, and a residue. The sifting process described as follows leads to extract each IMF and the residue.

Initially, the first residue and the first difference take the value of the initial EEG data.

- Step1: Locate the local maxima and minima of the difference.

- Step2: Calculate the lower and the upper envelop using these extrema.

- Step3: Calculate the mean by averaging the upper and the lower envelop. 
- Step4: Calculate the new difference by subtracting the mean from the previous difference.

- Step5: If the stopping criteria are satisfactory, then the last calculated difference is an IMF, otherwise go to step 1 and continue the process step by step.

- Step6: Calculate the new residue by subtracting the last IMF from the previous residue.

- Step7: If the new residue is not isometric then repeat the process from step1. Otherwise, the sifting process is ended, and the last found residue is considered as the final residue of the process.

The stopping criteria are a compound of two conditions. First, the number of extrema and the number of zero crossing must be either equal or differ by one at most. Second, the standard deviation (SD) is smaller than a predetermined value.

$$
S D=\frac{\sum_{t=0}^{T}\left|h_{k-1}(t)-h_{k}(t)\right|^{2}}{\sum_{t=0}^{T} h_{k-1}^{2}(t)}
$$

Where: $h_{i}$ are the vectors of differences; $k$ is the differences counter and $T$ is the period of considered samples.

Figure 4 illustrates the result of the sifting process for a SSVEP signal during the period of $2 \mathrm{~s}$.

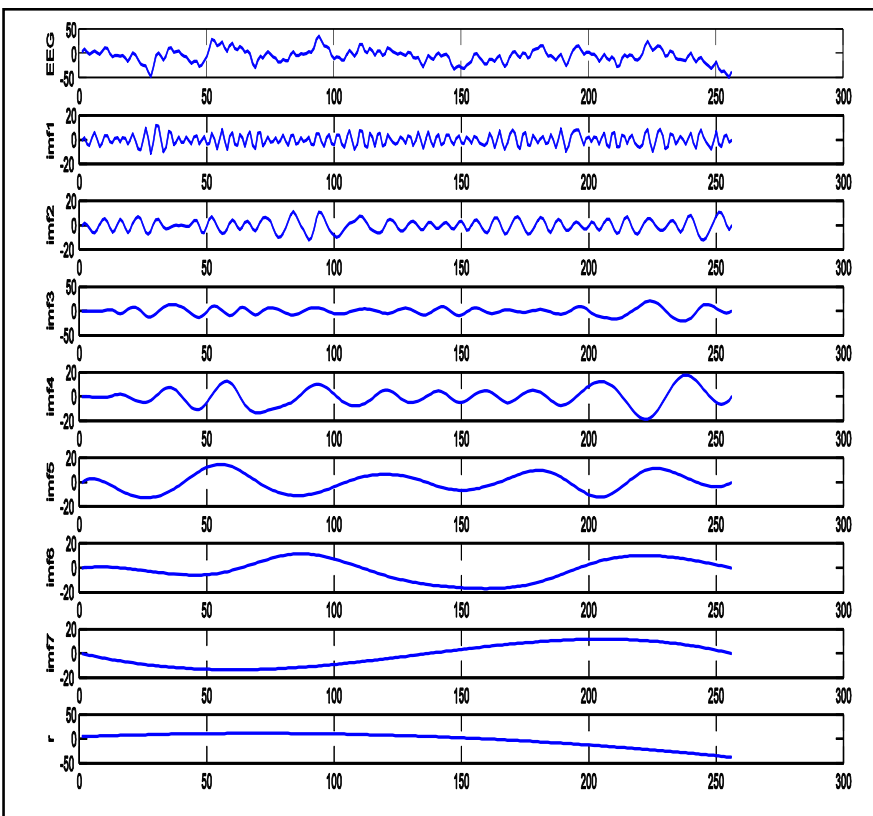

Fig. 4. Decomposition of the original signal in IMFs and a residue.

Each IMF has a higher frequency than the next extracted one. The residual which is the lowest frequency component represents the trend of the signal.

The spectral analysis of the IMFs allows to separate them into three groups. The first contains the IMFs located in a lower frequency band than the stimulus frequency. This frequency and its neighbors constitute the frequency band of the second group. The last one contains the IMFs characterized by several scattered frequencies higher than the stimulation frequency.

Figures 5 (A)-(B) illustrate the Normalized Amplitude Spectrum (NAS) of different IMFs, defined in equation (10).

$$
N S A(x)=\frac{F F T(x)}{\sum_{i=1}^{M} F F T\left(x_{i}\right)}
$$

Where: $x$ is an IMF time series, $F F T(x)$ is the fast Fourier transform of $x$, and $M$ is the number of FFT points.

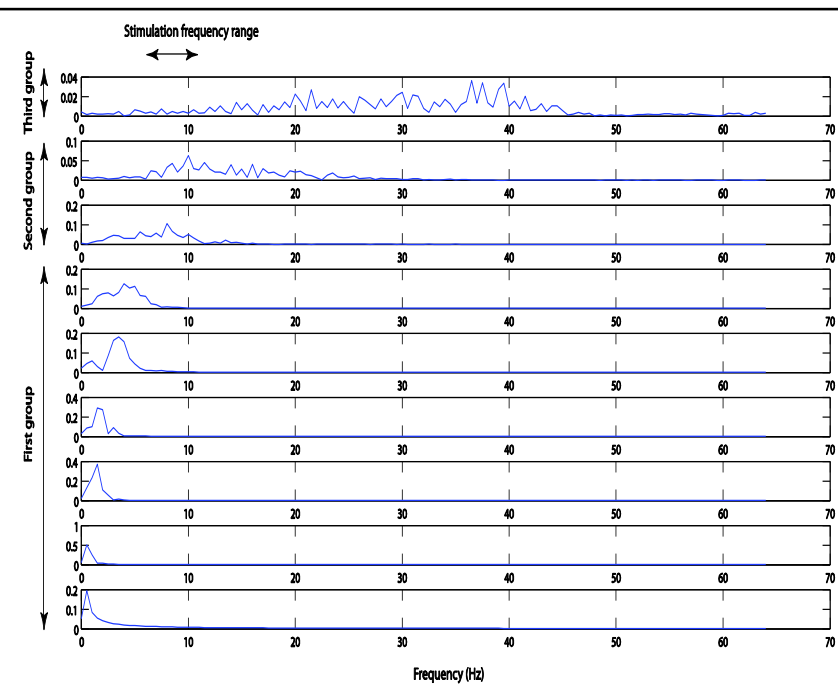

(a)

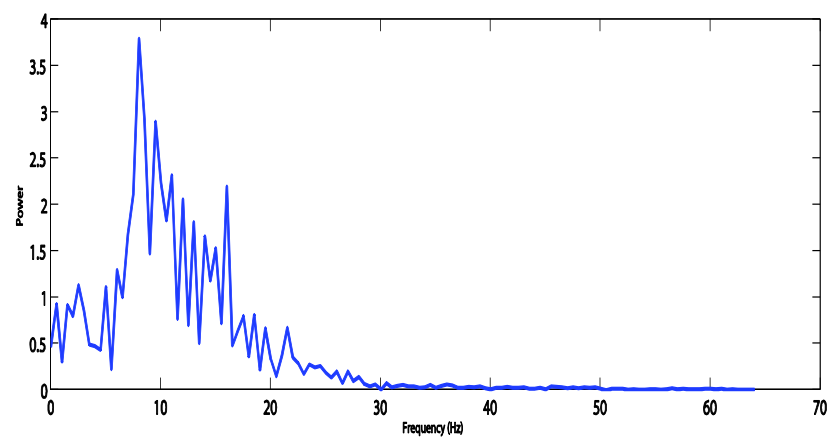

(b)

Fig. 5. Spectral analysis of the IMFs: (A) separation between the three groups, (B) spectrum of the stimulation frequency group.

The IMFs located in the first group are considered as noise containing neither stimulation frequencies nor harmonics. To discriminate between the first and the second group, a decision-making criterion is required. R. Sharma et al. [21] have used the sample entropy (SampEn) as a complexity measure of IMFs extracted from EEG signal. This experiment 
shows that the SampEn decreases from one IMF to the next. Therefore, SampEn can be a good criterion to separate the IMFs of the first group from the IMFs of the second. Likewise, IMFs which have a SampEn inferior to a predetermined threshold are considered as noise components.

After subtracting the IMFs of the first group from the original signal, the remainder can be considered as interesting or a mixture between the noise and the interesting signal according to the level of noise. The central frequency of the second IMF is in the stimulus frequencies band. Its NAS indicates the noise level. If the NAS is superior to a predetermined threshold, the signal to noise ratio is high enough and the first step of the MEC method is ended. However, in the second case, a filtering is needed to exclude the rest of noise while keeping the sharpest EEG signal response. The moving average filter is optimal for this kind of issue. In spite of its simplicity, it ensures a low curve shape change to keep valid the previous decompositions. To produce each point, some input points are averaging according to the equation (11).

$$
y_{i}=\frac{1}{M} \sum_{j=0}^{M-1} x_{i+j}
$$

Where $x_{i}$ is an input point, $y_{i}$ is an output point, and $M$ is the number of points in the average.

When $M$ increases the noise decreases but the acute curve angles become obtuse. The best choice of $M$ is about eleven [22].

\section{RESULTS AND DISCUSSION}

For the comparison purpose, the previously presented methods were implemented as well as the MEC proposed amelioration. The experiment results allow to validate the amelioration.

\section{A. Separation between noise and interesting signal}

The SampEn calculates the probability that epochs of window length $\mathrm{m}$ that are similar within a tolerance $\mathrm{r}$ remain similar at the next point [23]. A study of the different possible combinations shows that the best values of $m$ and $r$ are 6 and 0.2 respectively. Also, the best choices of the thresholds are 0.1 for the SampEn and 0.08 for the NAS.

Figure 6 reports an example of the extracted noise and interesting signal from the EEG recording. In the interesting signal, the stimulus frequency dominates the harmonics frequencies as it has a higher amplitude response than them [8]. Moreover, it follows the shape of the EEG signal i.e. the original features are maintained. The Nuisance signal frequency seems to be higher than the interesting signal as it includes the artifacts.

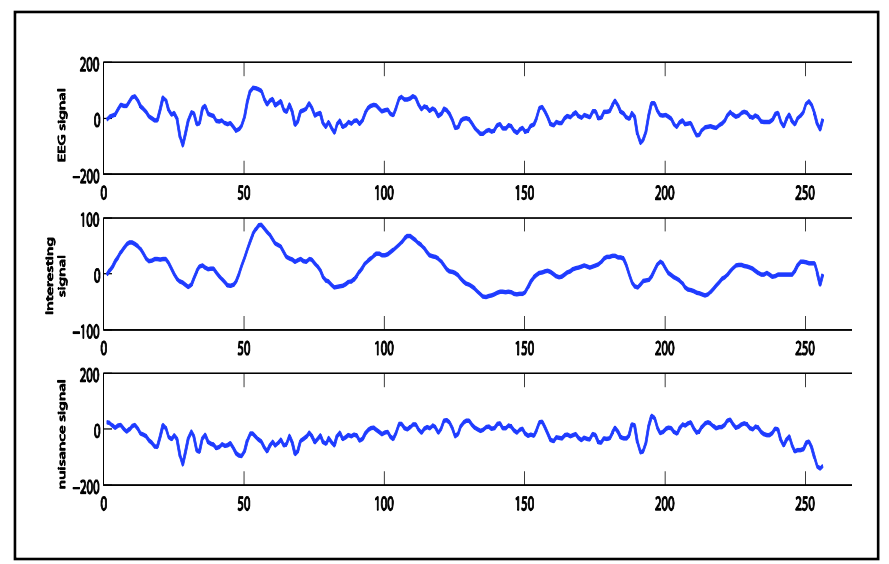

Fig. 6. Extracted noise and interesting signal using the proposed improvement.

\section{B. Stimuli color effect on different recognition methods}

Overlapping over several recording intervals can lead to improve the precision of the system. In this experiment, five intervals of a length of $3 \mathrm{~s}$ each and with a gap of $0.25 \mathrm{~s}$ were used. The idle period is fixed to $4 \mathrm{~s}$. The experiment consists of changing the colors of the three used LEDs. The colors are white, green, red, blue, and yellow. Table 1 illustrates the impact of the colors on the system accuracy rates using the processing methods mentioned before. The results show that the responses of white and yellow colors evoked an accuracy exceeding the $80 \%$. However, the precisions with red, green, or blue stimuli are lowest.

The white and the yellow color are the brightest which explains this result. In fact, D. G. Albrecht et al. [25] examined the effect of the contrast intensity of the visual stimulation on neurons from the Visual area one V1 or the striate cortex. These neurons are sensitive to the object features at which the subject gazes as the color and the direction. The results show that the response of a striate cell increases as the contrast intensifies. The Accuracy rate using a white color is most stable and high. This consequence justifies the choice of the white color for the next experiments.

TABLE I. SSVEP RESPONSES TO STIMULI OF DIFFERENT COLORS

\begin{tabular}{|c|c|c|c|c|}
\cline { 3 - 5 } \multicolumn{2}{c|}{} & \multicolumn{3}{c|}{ Method } \\
\cline { 3 - 5 } \multicolumn{2}{c|}{} & MEC & MSI & $\boldsymbol{C C A}$ \\
\hline \multirow{4}{*}{ Color } & Yellow & $44 \%$ & $89 \%$ & $72 \%$ \\
\cline { 2 - 5 } & Blue & $50 \%$ & $55 \%$ & $55 \%$ \\
\cline { 2 - 5 } & Green & $67 \%$ & $78 \%$ & $78 \%$ \\
\cline { 2 - 5 } & Red & $50 \%$ & $67 \%$ & $67 \%$ \\
\cline { 2 - 5 } & White & $72 \%$ & $83 \%$ & $83 \%$ \\
\hline
\end{tabular}

C. Noise level effects on different recognition methods

As explained before, the nature of the light source has a great effect on the noise level. In the next experiment, two kinds of white light LEDs are used in order to verify the noise sensitivity of each method. The first is the universal LEDs with $3 \mathrm{~mm}$ and the second is the chips on board (COB) LEDs. COB LEDs embedded ten LEDs in a circular surface with a diameter of $35 \mathrm{~mm}$. Therefore they can produce a sufficient light intensity without causing fatigue or losing the subject concentration. The subjects were asked to follow the scenario 
of the previous experiment. Figure 7 presents the accuracy rates of MEC, CCA, and MSI methods for the five subjects with the $3 \mathrm{~mm}$ LEDs. It is clear that the high level of noise leads to decrease the performances of all processing methods.

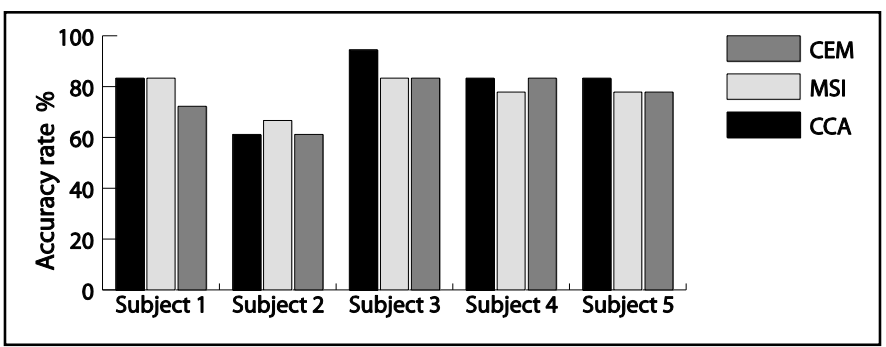

Fig. 7. SSVEP responses to $3 \mathrm{~mm}$ LEDs stimuli with different processing methods.

Table 2 illustrates the percentage of correct recognitions for each method using $3 \mathrm{~mm}$ LEDs.

TABLE II. AVERAGE ACCURACY OF EACH METHOD WITH 3MM LEDS

\begin{tabular}{|c|c|c|c|}
\hline Method & MEC & MSI & CCA \\
\hline $\begin{array}{c}\text { Accuracy } \\
\text { rate }\end{array}$ & $75.55 \%$ & $77.77 \%$ & $81.1 \%$ \\
\hline
\end{tabular}

The MEC responses remain the lowest even if the accuracy values are close.

In the next section, The COB LEDs replace the universal ones. Figure 8 illustrates the results of different methods.

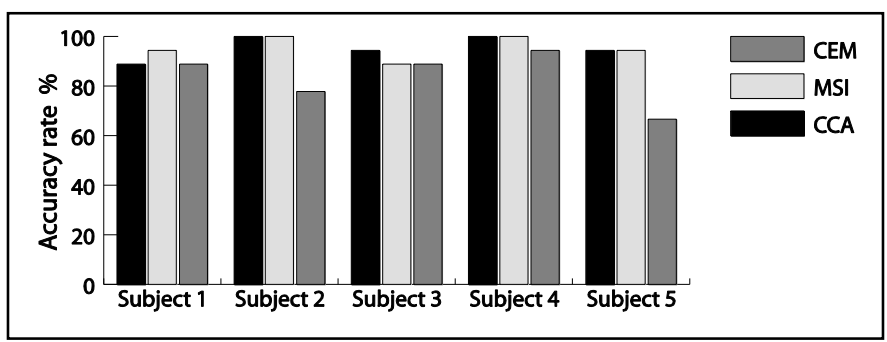

Fig. 8. The accuracy values with COB LEDs stimuli.

The experimental results show that all methods performed better using COB LEDs than using 3mm LEDs. Thus, the level of noise decreases using COB LEDs.

Table 3 illustrates the percentage of correct recognitions for each method using COB LEDs stimuli.

TABLE III. AVERAGE ACCURACY OF EACH METHOD WITH COB LEDS

\begin{tabular}{|c|l|l|l|}
\hline Method & MEC & MSI & CCA \\
\hline $\begin{array}{c}\text { Accuracy rate } \\
\text { (COB LED) }\end{array}$ & $86 \%$ & $95.55 \%$ & $95.55 \%$ \\
\hline $\begin{array}{c}\text { Amelioration value } \\
\text { (\%) }\end{array}$ & $10.28 \%$ & $22.85 \%$ & $17.8 \%$ \\
\hline
\end{tabular}

Although the three methods are noise sensitive, MEC remains largely poorer. This method is influenced even with little noise levels.

\section{Application of the proposed improvement}

The goal of this experiment is to validate the proposed improvement procedure of MEC method for the SSVEP features recognition. The COB LEDs are the light sources. The study of the average accuracy rates of MEC, CCA, and MSI methods allows to compare their noise sensitivities as well as the improved MEC for the five subjects. Three data intervals of $2 \mathrm{~s}$ with a gap of $0.25 \mathrm{~s}$ were used to identify the target.

Table 4 summarizes the performances of the different methods.

TABLE IV. RECOGNITION METHODS PERFORMANCE

\begin{tabular}{|c|c|c|c|c|}
\hline Method & CCA & MSI & MEC & $\begin{array}{c}\text { Improved } \\
\text { MEC }\end{array}$ \\
\hline $\begin{array}{c}\text { Accuracy } \\
\text { rate }\end{array}$ & $96.66 \%$ & $96.66 \%$ & $83.33 \%$ & $98.88 \%$ \\
\hline
\end{tabular}

The proposed improvement produces more precise results in selecting the stimulation frequency. It ameliorates the accuracy of the MEC method by about $13 \%$. Thus, MEC becomes efficiently resistant to the noise. This method becomes even more performant than other methods and reaches $99 \%$ with a total recording interval of $2.5 \mathrm{~s}$. This investigation confirms the hypothesis that is using the EMD and the moving average filter allows to separate noise and artifact from the interesting signal. EMD decomposes a nonlinear and nonstationary signal into a sum of IMFs without the need of prior knowledge. In fact, it is an adaptive technique depending on the local characteristic of the signal which explains its compliance with the moving average filter. The results confirm the validity of the improvement.

\section{E. Information-transfer rate}

One of the most used metrics to evaluate the performance of BCI systems is the information-transfer rate (ITR). Wolpaw et al. [26] have proposed the most popular method for ITR calculation as defined in equation (12).

$B=\log _{2} N+P \log _{2} P+(1-P) \log _{2}[(1-P) /(N-1)]$

Where $\mathrm{B}$ is the ITR (bits/symbol), $\mathrm{N}$ is the number of possible commands and $\mathrm{P}$ is the classification accuracy. In order to make this quantity easier to understand, another ITR definition $B_{t}$ in bits/min which is derived from B is generally used.

$$
B_{t}=B^{*}(60 / T)
$$

Where $\mathrm{T}$ is the average time needed to convert a brain feature activity into a command.

A higher ITR leads to a better and more natural use of the system. In fact, this criterion reflects the time during which the subject has to gaze at the target and the number of commands needed to reach the destination. In order to foster this criterion, another light source was added to the stimulation system. Also, 
the idle period and the trial length have gradually been reduced. Moreover, in order to reduce the number of needed time intervals, a decision is made as soon as the same target is identified three times by the system in which the total number of intervals is equal or less than five. Otherwise, if five-time intervals are processing without recognizing the target, the trial is considered as erroneous. Table 5 illustrates a comparison of the CCA method and the proposed method where the idle period is equal to $0.5 \mathrm{~s}$ and the interval is equal to $2 \mathrm{~s}$. A lower interval decreases enormously the accuracy of the system and obviously increases the time cost needed to fix the wrong choices.

TABLE V. MEAN ITR AND ACCURACY AS A FUNCTION OF NUMBER OF TARGETS FOR CCA AND IMPROVED MEC METHODS

\begin{tabular}{|c|c|c|c|c|}
\cline { 2 - 5 } \multicolumn{1}{c|}{} & \multicolumn{4}{c|}{ Method } \\
\cline { 2 - 5 } \multicolumn{1}{c|}{} & \multicolumn{2}{c|}{ Improved MEC } & \multicolumn{2}{c|}{ CCA } \\
\cline { 2 - 5 } \multicolumn{1}{c|}{} & $\mathbf{3}$ targets & 4 targets & 3 targets & 4 targets \\
\hline $\begin{array}{c}\text { Average } \\
\text { accuracy } \\
\text { rate }\end{array}$ & $95.5 \%$ & $91.78 \%$ & $91.11 \%$ & $88.14 \%$ \\
\hline $\begin{array}{c}\text { Average } \\
\text { trial length }\end{array}$ & $3.108 \mathrm{~s}$ & $3.22 \mathrm{~s}$ & $3.046 \mathrm{~s}$ & $3.133 \mathrm{~s}$ \\
\hline $\begin{array}{c}\text { Average } \\
\text { informatio } \\
\text { n-transfer } \\
\text { rate } \\
\text { (bits/min) }\end{array}$ & 24.617 & 27.18 & 20.944 & 24.64 \\
\hline
\end{tabular}

The results of this experiment show that the proposed method remains better even with the new scenario. The system can reach acceptable performances with four commands.

\section{CONCLUSION}

Steady State Visual Evoked Potential is the most effective solution for BCIs in everyday use. Its low required training and high accuracy rate make its use close to the ordinary one. Three LEDs with different frequencies $(8,9$ and $10 \mathrm{~Hz}$ ) were used during the first experiments. Later an additional LED flickers at $11 \mathrm{~Hz}$ was added. Each LED represents a possible direction to control the navigation of an electric wheelchair. The EPOC EMOTIVE headset was used to acquire data from the five volunteers. Only the closest six electrodes to the occipital area were used. In this study, a new amelioration to improve the robustness against the noise of the Minimum Energy Combination method was proposed. Results prove that the stimulus characteristics have a great impact on the noise level in the SSVEP signal. The use of COB LEDs allows to increase the SNR. Also the white color increases the excitation of neurons from the visual cortex of the brain and allows to reach the best accuracy values. The Canonical Correlation Analysis and the Multivariate Synchronization Index based methods serve as references for comparing their performances with the performances of the proposed improvement. The results indicated that the improved MEC method performed better than the widely used CCA and MSI. The average accuracy rate reaches $99 \%$ and increases by about $13 \%$ compared to the original MEC with a data length of $2.5 \mathrm{~s}$ using three targets. Also using four targets, the system reached an average information-transfer rate of about $27.5 \mathrm{bits} / \mathrm{min}$. This makes the system more suitable for the wheelchair navigation command.

\section{REFERENCES}

[1] A. L. S. Ferreira, L. C. de Miranda, E. E. C. de Miranda and S. G. Sakamoto "A Survey of Interactive Systems based on Brain-Computer Interfaces", SBC Journal on 3D Interactive Systems, VOL. 4, NO. 1, 2013, pp 3-13.

[2] M. Middendorf, G. McMillan, G. Calhoun and K. S. Jones "BrainComputer Interfaces Based on the Steady-State Visual-Evoked Response", IEEE Transactions on Rehabilitation Engineering, VOL. 8, NO. 2, 2000, pp 211-214.

[3] W. Yijun, W. Ruiping, G. Xiaorong and G. Shangkai "Brain-computer Interface based on the High-frequency Steady-stateVisual Evoked Potential", First International Conference on Neural Interface and Control Proceedings, Wuhan, China, 26-28 May 2005, pp 37-39.

[4] Y. Wang, R. Wang, X. Gao, B. Hong and S. Gao "A Practical VEPBased Brain-Computer Interface", IEEE Transactions on neural systems and rehabilitation engineering, VOL. 14, NO. 2, 2006, pp 234-239.

[5] F. Gembler, P. Stawicki and I. Volosyak "A Comparison of SSVEPBased BCI-Performance Between Different Age Groups", 13th International Work-Conference on Artificial Neural Networks, Palma de Mallorca, Spain, June 10-12 2015, pp 71-77.

[6] Q. Liu, K. Chen, Q. Ai and S. Q. Xie "Review: Recent Development of Signal Processing Algorithms for SSVEP-based Brain Computer Interfaces", Journal of Medical and Biological Engineering, VOL. 34, NO. 4, 2013, pp 299-309.

[7] O. Friman, I. Volosyak and A. Gräser "Multiple Channel Detection of Steady-State Visual Evoked Potentials for Brain-Computer Interfaces", IEEE transactions on biomedical engineering, VOL. 54, NO. 4, APRIL 2007, pp 742-750.

[8] N. Chumerin, N. V. Manyakov, A. Combaz, Ar. Robben, M. vanVliet and M. M. Van Hulle "Steady State Visual Evoked Potential Based Computer Gaming - The Maze", Lecture notes of the Institute for Computer Sciences, Social Informatics and Telecommunications Engineering, INTETAIN 2011, LNICST VOL. 78, 2012, pp. 28-37.

[9] Z. Lin, C. Zhang, W. Wu and X. Gao "Frequency Recognition Based on Canonical Correlation Analysis for SSVEP-Based BCIs", IEEE transactions on biomedical engineering, VOL. 54, NO. 6, JUNE 2007, pp 1172-1176.

[10] G. Hakvoort, B. Reuderink and M. Obbink "Comparison of PSDA and CCA detection methods in a SSVEP-based BCI-system", technical report TR-CTIT-11-03, center for telematics and information technology, university of twente (2011).

[11] N. Mora, V. Bianchi, I. De Munari and P. Ciampolini "A BCI Platform Supporting AAL Applications", 8th International Conference Universal Access in Human-Computer Interaction, Heraklion, Crete, Greece, June 22-27 2014, pp. 515-526.

[12] W. Nan, C. M. Wong, B. Wang, F. Wan, P. U. Mak, P. I. Mak and M. Vai "A Comparison of Minimum Energy Combination and Canonical Correlation Analysis for SSVEP Detection", the 5th International IEEE EMBS Conference on Neural Engineering Cancun, Mexico, April 27 May 1 2011, pp 469-472.

[13] Y. Zhang, P. Xu, K. Cheng and D. Yao "Multivariate synchronization index for frequency recognition of SSVEP-based brain-computer interface", Journal of Neuroscience Methods, VOL. 221, 15 January 2014, pp 32- 40.

[14] C. S. Herrmann "Human EEG responses to 1-100 Hz flicker: resonance phenomena in visual cortex and their potential correlation to cognitive phenomena", Experimental Brain Research, NO. 137, Springer-Verlag, 2001, pp 346-353.

[15] F.C. Lin, J. K. Zao, K.C. Tu, Y. Wang, Y.P. Huang, C.W. Chuang, H.Y. Kuo, Y.Y. Chien, C.-C. Chou and T.P. Jung "SNR Analysis of HighFrequency Steady-State Visual Evoked Potentials from the Foveal and Extrafoveal Regions of Human Retina", 34th Annual International Conference of the IEEE Engineering in Medicine and Biology Society 
EMBS, San Diego, California USA, 28 August - 1 September 2012, pp 1810-1814.

[16] E. E. Sutter "The brain response interface: communication through visually-induced electrical brain responses", Journal of Microcomputer Applications VOL. 15, issue 1, 1992, pp 31-45.

[17] A. González-Mendoza, J. L. Pérez-Benítez, J. A. Pérez-Benítez and J.H. Espina-Hernández "Brain Computer Interface based on SSVEP for controlling a remote control car", International Conference on Electronics, Communications and Computers (CONIELECOMP), Cholula, 25-27 Feb. 2015, pp 93-97.

[18] A. Duszyk, M. Bierzynska, Z. Radzikowska, P. Milanowski, R. Kus, P. Suffczynski, M. Michalska, M. Labecki, P. Zwolinski and P. Durka "Towards an Optimization of Stimulus Parameters for Brain-Computer Interfaces Based on Steady State Visual Evoked Potentials," Plos One, VOL. 9, NO. 11, 2014, pp.11.

[19] Y. Liu, X. Jiang, T. Cao, F. Wan, P. U. Mak, P.-I. Mak and M. I Vai "Implementation of SSVEP Based BCI with Emotiv EPOC", IEEE International Conference on Virtual Environments Human-Computer Interfaces and Measurement Systems (VECIMS), Tianjin, 2-4 July 2012, pp 34-37.

[20] F. Lotte, M. Congedo, A. Lécuyer, F. Lamarche and B. Arnaldi. "A review of classification algorithms for EEG-based brain-computer interfaces," Journal of Neural Engineering, IOP Publishing, 2007, 4, pp.24.

[21] N. E. Huang, Z. Shen, S. R. Long, M. C. Wu, H. H. Shih, Q. Zheng, N.C. Yen, C. C. Tung and H. H. Liu "The empirical mode decomposition and the Hilbert spectrum for nonlinear and non-stationary time series analysis", Proc. The Royal Society, Lond. A (1998) 454, pp 903-995, Printed in Great Britain.

[22] R. Sharma, R. BilasPachori and U. R. Acharya "Application of Entropy Measures on Intrinsic Mode Functions for the Automated Identification of Focal Electroencephalogram Signals", Entropy, NO. 17, 2015, pp 669-691.

[23] S. Smith "Digital Signal Processing: A Practical Guide for Engineers and Scientists, 1stEdition" 07 Nov. 2002 pp. 279-280.

[24] D. E. Lake, J. S. Richman, M. P. Griffin and J. Randall Moorman "Sample entropy analysis of neonatal heart rate variability", American Journal of Physiology Regulatory, Integrative and Comparative Physiology, 1 September 2002 Vol. 283 no. 3, R789-R797.

[25] D. G. ALBRECHT and D. B. HAMILTON "Striate Cortex of Monkey and Cat: Contrast Response Function" journal of neurophysiology, Vol.48, No.1, 1982.

[26] J. R. Wolpaw, H. Ramoser, D. J. McFarland, and G. Pfurtscheller "EEG-Based communication: improved accuracy by response verification" IEEE TRANSACTIONS ON REHABILITATION ENGINEERING, VOL. 6, NO. 3, SEPTEMBER 1998, pp. 326-33. 